\title{
Electric field-dependent dynamic polarizability and state-insensitive conditions for optical trapping of diatomic polar molecules
}

\author{
Svetlana Kotochigova ${ }^{1 *}$ and David DeMille ${ }^{2}$ \\ ${ }^{1}$ Department of Physics, Temple University, Philadelphia, PA 19122-6082, USA \\ ${ }^{2}$ Department of Physics, Yale University, New Haven, CT 06520, USA
}

\begin{abstract}
Selection of state-insensitive or "magic" trapping conditions with ultracold atoms or molecules, where pairs of internal states experience identical trapping potentials, brings substantial benefits to precision measurements and quantum computing schemes. Working at such conditions could ensure that detrimental effects of inevitable inhomogeneities across an ultracold sample are significantly reduced. However, this aspect of confinement remains unexplored for ultracold polar molecules. Here, we present means to control the AC Stark shift of rotational states of ultracold diatomic polar molecules, when subjected to both trapping laser light and an external electric field. We show that both the strength and relative orientation of the two fields influence the trapping potential. In particular, we predict "magic electric field strengths" and a "magic angle", where the Stark shift is independent of the DC external field for certain rotational states of the molecule.
\end{abstract}

The advantage of using state-independent light traps for precision frequency measurements with ultracold atoms has been demonstrated in several experiments [1, 2]. Applications of this approach were analyzed in the context of optical atomic clocks and coherent control of atoms and photons within an optical cavity. For these applications the frequency of the laser beam that creates a far-off resonant optical dipole trap is chosen such that the AC Stark shift of the ground and one excited electronic atomic level are the same. In this way any optical atomic transition between these levels is unaffected by the trapping light. In Refs. [3 6] state-insensitive trapping conditions have also been found for microwave transitions in the atomic ground state by using a combination of the vector and tensor components of the AC Stark shift and an external magnetic field.

Ultracold molecular systems possess unique proporties that are considered to make them potentially useful as tools for precision measurements [7, 8] and quantum computing [9, 10]. Therefore it is desirable to extend the zero-differential AC Stark shift technique to these more complex systems. The idea of "magic" frequencies for vibrational Raman transitions in homo-nuclear $\mathrm{Sr}_{2}$ molecules was first explored in Refs. [11, 12. This molecule is proposed for a search of possible time variation of the electron-to-proton mass ratio. The AC Stark shift of a molecule is determined by the dynamic molecular polarizability $\alpha(\nu)$, which is a function of radiation frequency $\nu$ and its polarization.

Polar molecules have a permanent dipole moment and their levels can be shifted and mixed with one another by applying an external electric field. This opens up a new way to create "magic" trapping conditions for two rotational levels of the molecule. In the presence of an external electric field, $J$ is not a good quantum number and all states, even the "rotationless" ground state, have an anisotropic polarizability [13]. The anisotropy of 
the dynamic polarizability of these levels manifests itself as a dependence on the relative orientation of the polarization of the trapping laser and the DC electric field. The combined action of these two fields can be a powerful tool to manipulate and control ultracold molecules trapped in an optical potential.

The behavior described here has potential applications to several experiments that have been envisioned for diatomic polar molecules held in optical traps. The implications are particularly striking for the use of polar molecules in an optical lattice as quantum bits. As was described in Ref. [9], a pair of rotational states forms a suitable quantum bit. However, it was pointed out that this system is susceptible to decoherence due to intensity fluctuations in the optical trapping lasers, if the dynamic polarizabilities of these levels differ as is generally the case. This in turn leads to very stringent requirements on the laser intensity stability for such a system to be practical. As we will show, this limitation can be removed by adjusting the experimental parameters to guarantee that the dynamic polarizabilities of these states are equal. In particular, for this proposed system (where a spatial gradient of the electric field $\overrightarrow{\mathcal{E}}$ is required, so working at a magic electric field value is impossible) it should be possible to use light polarized at the "magic angle" relative to the static field in order to eliminate this potentially dangerous source of decoherence.

The existence of magic electric field values is also of possible use for envisioned applications where polar molecules in optical lattices are employed in novel types of many-body systems. This includes, for example, cases where the properties of long-range molecular interactions are tailored by a combination of a static electric field and resonant microwave fields coupling different rotational states [14-16. Working at a magic electric field value in such systems could ensure that the inevitable inhomogeneities in the intensity of the trapping light across a large sample would not change the resonant condition for the microwave drive fields. Hence, working under such "magic" conditions might be necessary to implement proposals of this type.

Motivated by these ideas, we calculate the near-infrared dynamic polarizability of various rotational levels of the $v=0$ vibrational state of the $\mathrm{X}^{1} \Sigma^{+}$potential of the $\mathrm{KRb}$ and RbCs molecules, under the simultaneous influence of trapping electromagnetic and static electric fields. We calculate dynamic polarizability using the computational techniques developed in previous publications [17-19]. Our calculations with an external electric field are predominantly performed at a laser frequency of $9174 \mathrm{~cm}^{-1}$ (or wavelength of $1090 \mathrm{~nm}$ ), which corresponds to an often-used frequency to trap atoms and molecules in ultracold experiments. In the near infrared this laser frequency is sufficiently far away from molecular resonances of the excited electronic states that heating due to photon scattering is negligible. We focus on external electric field strengths up to $15 \mathrm{kV} / \mathrm{cm}$, a value which should be experimentally accessible.

We begin by studying the dynamic polarizability or AC Stark shift for the $J=0$ and $J=1$ rotational levels of the $v=0 \mathrm{X}^{1} \Sigma^{+}$state of $\mathrm{KRb}$ and RbCs in the near infrared, without an external electric field. The procedure used to determine the complex molecular polarizability has already been described in our previous paper [17]. Figure 1]illustrates the results of this study in the absence of an electric field by showing the dynamic polarizability $\alpha$ as a function of laser frequency $h \nu$. The range of laser frequencies spans a technologically relevant near-infrared optical domain and includes the lasing frequency near $9174 \mathrm{~cm}^{-1}$ used in Ref. [13. The figure also shows that the dynamic polarizability of the molecular state depends on the rotational quantum number $J$ and its projection $M$. The curves for 


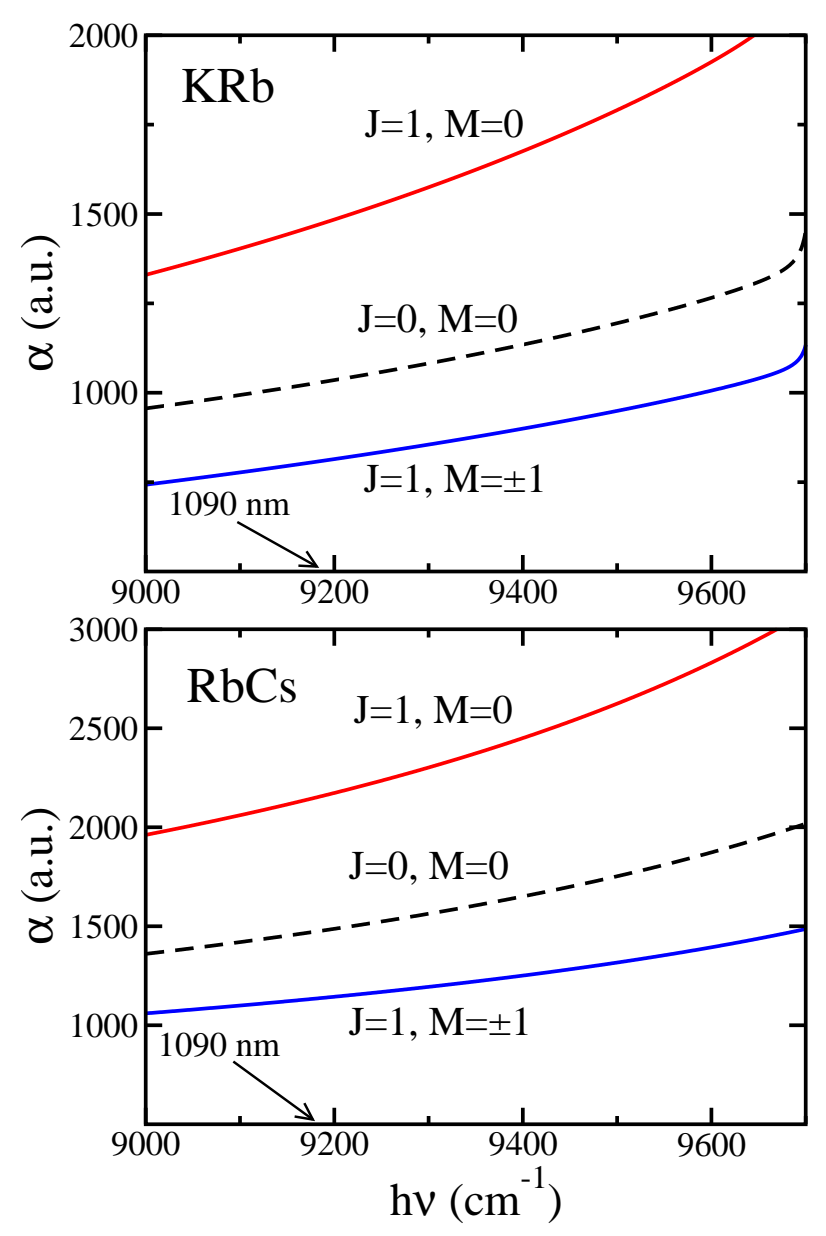

FIG. 1: Dynamic polarizability in the absence of an electric field and in atomic units for the $v=0$, $J=0$ and 1 levels of the $\mathrm{X}^{1} \Sigma^{+}$ground state of $\mathrm{KRb}$ and RbCs as a function of trapping frequency in the near infrared domain. The dashed lines show the polarizability for the $J=0$ and $M=0$ level, which is independent of the light polarization. The solid lines correspond to the $J=1$ state with magnetic sublevels $M=0$ and \pm 1 illuminated by linear polarized light along the $\hat{z}$ direction. One atomic unit of polarizability corresponds to $4.68645 \times 10^{-8} \mathrm{MHz} /\left(\mathrm{W} / \mathrm{cm}^{2}\right)$.

the different states do not cross as a function of the laser frequency. In other words, at zero electric field we can not find a "magic" frequency for $\mathrm{J}=0$ and $\mathrm{J}=1$ states using trapping light in the near infrared.

\section{Field-dependent dynamic polarizability}

Here we extend the idea of the AC Stark shift for the rotational levels of the $\mathrm{X}^{1} \Sigma^{+}$ground state to the mixing of these levels in a static electric field $\overrightarrow{\mathcal{E}}=\mathcal{E} \hat{z}$ along the space-fixed $\hat{z}$ direction. Our analyses show that the experimentally accessible electric fields mix only a few low-lying rotational states. The ultimate goal is to introduce the dynamic polarizability of the mixed rotational levels as a function of an external DC electric field and the polarization of the AC trapping field. In a heteronuclear molecule this Stark mixing is primarily due to the permanent electronic dipole moment $\vec{d}$; we neglect the much smaller effects due to 
Stark mixing with other electronic states. For the Hund's case (a) $\mathrm{X}^{1} \Sigma^{+}$state of an alkalimetal dimer, the total electron spin and orbital angular momentum are not coupled to the molecular rotation. The molecular wavefunction in the lab frame, $|v J M\rangle_{z}$, is then given by

$$
|v J M\rangle_{z} \equiv|v J\rangle \times Y_{J M}(\hat{R})=\left\{\frac{\psi_{v J}(R)}{R}\left|\mathrm{X}^{1} \Sigma^{+}\right\rangle\right\} \times Y_{J M}(\hat{R}),
$$

where $v$ is a vibrational quantum number, $J$ and $M$ are the molecular rotational angular momentum and its projection along the $z$ axis, $\psi_{v J}(R)$ is the radial rovibrational wavefunction, $Y_{J M}(\hat{R})$ is a spherical harmonic, $\hat{R}$ is the orientation of the molecule relative to the electric field direction $z,\left|\mathrm{X}^{1} \Sigma^{+}\right\rangle$is the electronic wavefunction with projections defined along the internuclear axis. For the $\mathrm{X}^{1} \Sigma^{+}$state, in even modest electric fields the nuclear spins are well-decoupled from the other spins and angular momenta; hence we ignore the nuclear spins here. Then the Hamiltonian for such a system becomes

$$
H=\sum_{v J M} E_{v J}|v J M\rangle_{z} \times{ }_{z}\langle v J M|-\vec{d} \cdot \overrightarrow{\mathcal{E}}
$$

where $E_{v J}=G_{v}+B_{v} J(J+1)$ and $G_{v}$ and $B_{v}$ are the vibrational energy and rotational constant of vibrational level $v$, respectively. Higher order rotational corrections are negligible. For the alkali-metal dimers KRb and RbCs we have that $\Delta G_{v}=G_{v+1}-G_{v}$ is on the order of $50-100 \mathrm{~cm}^{-1}$ for small $v$, while $B_{v}$ is on the order of $0.017-0.037 \mathrm{~cm}^{-1}$.

We evaluate the matrix elements of the operator $-\vec{d} \cdot \overrightarrow{\mathcal{E}}$ by noting that after averaging over the electronic wavefunction $\left|\mathrm{X}^{1} \Sigma^{+}\right\rangle$it reduces to $-d(R) C_{10}(\hat{R}) \mathcal{E}_{z}$, where $d(R)=\left\langle\mathrm{X}^{1} \Sigma^{+}|d| \mathrm{X}^{1} \Sigma^{+}\right\rangle$is the $R$-dependent permanent electric dipole moment, $C_{l m}(\hat{R})=$ $\sqrt{4 \pi /(2 l+1)} Y_{l m}(\hat{R})$ are tensors of $\operatorname{rank} l$, and $\mathcal{E}_{z}$ is the electric field strength. Consequently, this operator conserves the projection quantum number $M$. The matrix element between two rovibrational states is

$$
\begin{aligned}
{ }_{z}\langle v J M| & -\vec{d} \cdot \overrightarrow{\mathcal{E}}\left|v^{\prime} J^{\prime} M^{\prime}\right\rangle_{z}=-\delta_{M M^{\prime}} d_{v J, v^{\prime} J^{\prime}} \mathcal{E}_{z} \int d \hat{R} Y_{J M}^{*}(\hat{R}) C_{10}(\hat{R}) Y_{J^{\prime} M^{\prime}}(\hat{R}) \\
& =-\delta_{M M^{\prime}} d_{v J, v^{\prime} J^{\prime}} \mathcal{E}_{z}(-1)^{M} \sqrt{(2 J+1)\left(2 J^{\prime}+1\right)}\left(\begin{array}{ccc}
J & 1 & J^{\prime} \\
-M & 0 & M^{\prime}
\end{array}\right)\left(\begin{array}{ccc}
J & 1 & J^{\prime} \\
0 & 0 & 0
\end{array}\right),
\end{aligned}
$$

where $\delta_{M M^{\prime}}$ is the Kronecker delta function, $d_{v J, v^{\prime} J^{\prime}}=\int_{0}^{\infty} d R \psi_{v J}(R) d(R) \psi_{v^{\prime} J^{\prime}}(R)$, and $(\cdots)$ are $3-j$ symbols (see e.g. [20]). This matrix element is nonzero when $J+1+J^{\prime}$ is even, according to the parity selection rules, and is independent of the sign of $M$ and $M^{\prime}$. For the small $J$ values of interest here we can assume that the $J$ dependence of $\psi_{v J}(R)$ is negligible. Moreover, coupling between vibrational levels can also be ignored, as the dipole moment $d(R)$ is a slowly varying function with $R$ and the spacing between vibrational levels is large compared to the rotational splitting. For tensor operators $C_{l m}(\hat{R})$ of rank 0,1 , and 2 the relationship between Cartesian $x, y$, and $z$ components and spherical $m=-1$, 0 , and +1 components can be found in Refs. [21, 22].

For each projection $M$ and vibrational level $v$, eigenvalues and eigenvectors of the Hamiltonian, Eq. (2), are obtained by the direct diagonalization of the Hamiltonian matrix including rotational states $J$ from $|M|$ up to some value $J_{\max }$. For the external electric field strength and $J$ values of interest, $J_{\max }=10$ is sufficient for convergence. We label the eigenenergies by $E_{v \tilde{J} M}$ with corresponding eigenvectors $|v \tilde{J} M\rangle=\sum_{J} U_{\tilde{J}, J}^{v M}|v J M\rangle_{z}$. 
Here $\tilde{J}$ is an integer index with values $\tilde{J}=|M|,|M|+1, \ldots$, such that the eigenstate $|v \tilde{J} M\rangle$ adiabatically connects to the electric field-free eigenstate $|v J M\rangle_{z}$ with $J=\tilde{J}$. For $|M|>0$ the levels with projection quantum number $-M$ and $M$ remain degenerate. The dipole matrix element between states $|v \tilde{J} M\rangle$ and $\left|v^{\prime} \tilde{J}^{\prime} M^{\prime}\right\rangle$ of the $\mathrm{X}$ potential is given by $\left\langle v \tilde{J} M\left|d_{\sigma}\right| v^{\prime} \tilde{J}^{\prime} M^{\prime}\right\rangle=\sum_{J, J^{\prime}} U_{\tilde{J}, J}^{v M} U_{\tilde{J}^{\prime}, J^{\prime}}^{v^{\prime} M^{\prime}} \times{ }_{z}\left\langle v J M\left|d_{\sigma}\right| v^{\prime} J^{\prime} M^{\prime}\right\rangle_{z}$, where $\sigma$ is a spatial index that can be expressed either in Cartesian coordinates $x, y, z$, or spherical coodinates $q=0, \pm 1$.

Now we are able to calculate the dynamic polarizability of the mixed rotational eigenstates at laser frequency $\nu$. It is determined by the properties of the operator $\alpha_{\sigma \sigma^{\prime}}(\nu)$ defined by [21, 23]

$$
\alpha_{\sigma \sigma^{\prime}}(\nu)=\sum_{\gamma}\left\{\frac{1}{E_{\gamma}-E_{v \tilde{J} M}+h \nu}+\frac{1}{E_{\gamma}-E_{v \tilde{J} M}-h \nu}\right\} d_{\sigma}|\gamma\rangle\langle\gamma| d_{\sigma^{\prime}}
$$

with $\gamma$ enumerating eigenstates of the ground as well as excited electronic potentials in the presence of the electric field and $\sigma, \sigma^{\prime}=x, y$, or $z$. We will focus on the $J=0$ and $J=1$ levels and without loss of generality assume that the $x$ axis of our coordinate system lies in the plane spanned by the electric-field direction and the orientation of the linearlypolarized laser light. Moreover, we are interested in the situation where the level shifts due to the laser are small compared to those induced by the electric field. For the isolated levels with $\mathrm{M}=0$, the polarizability is determined by the diagonal matrix element $\alpha_{\sigma \sigma^{\prime}}^{v \tilde{J} M=0}(\nu)=$ $\left\langle v \tilde{J} M=0\left|\alpha_{\sigma \sigma^{\prime}}(\nu)\right| v \tilde{J} M=0\right\rangle$. The $|M|>0$ dynamic polarizability needs to be treated by degenerate perturbation theory within the two-dimensional subspace $|v \tilde{J} M\rangle$ and $\mid v \tilde{J}-$ $M\rangle$. In fact, our choice of the $x$ direction and the symmetry properties of the dynamic polarizability ensure that the linear combinations $|v \tilde{J} M, \pm\rangle=\{|v \tilde{J} M\rangle \pm|v \tilde{J}-M\rangle\} / \sqrt{2}$ with $M>0$ are the correct eigenstates. For these states the dynamic polarizability $\alpha_{\sigma \sigma^{\prime}}^{v \tilde{J} M, \pm}(\nu)=$ $\left\langle v \tilde{J} M, \pm\left|\alpha_{\sigma \sigma^{\prime}}(\nu)\right| v \tilde{J} M, \pm\right\rangle$. For diatomic species, only the diagonal elements $\alpha_{x x}^{v \tilde{J} M, \pm}, \alpha_{y y}^{v \tilde{J} M, \pm}$, and $\alpha_{z z}^{v \tilde{J} M, \pm}$ are nonzero. Hence in an oscillating electric field $\overrightarrow{\mathcal{E}}_{o}(t)=\mathcal{E}_{o}(0) \operatorname{Re}\left\{\vec{\epsilon} e^{i 2 \pi \nu t}\right\}$ (where $\vec{\epsilon}$ is the complex unit vector indicating the polarization), the state $|v \tilde{J} M, \pm\rangle$ shifts in energy by an amount $\Delta E$ given by $\Delta E=-\sum_{\sigma, \sigma^{\prime}}\left|\mathcal{E}_{o}(0)\right|^{2} \alpha_{\sigma \sigma^{\prime}}^{v \tilde{J} M, \pm}(\nu) \epsilon_{\sigma} \epsilon_{\sigma^{\prime}}^{*} / 4$. The sum is over the spatial indices $\sigma, \sigma^{\prime}=x, y, z$.

We are interested in near infrared laser frequencies, which are detuned away from resonances with rovibrational levels of the electronically excited potentials. In particular, we focus on wavelengths between $1000 \mathrm{~nm}$ and $1100 \mathrm{~nm}$. Starting from the $v=0$ vibrational level of the $\mathrm{X}^{1} \Sigma^{+}$states of $\mathrm{KRb}$ and RbCs, photons of this wavelength do not possess sufficient energy to reach rovibrational levels of the electronically excited singlet ${ }^{1} \Lambda$ potentials. The photons do have enough energy to reach vibrational level of the triplet $b^{3} \Pi$ potential considering only single-photon excitations. However, such transitions require relativistic spin-orbit coupling to the $\mathrm{A}^{1} \Sigma^{+}$potential to acquire a non-zero dipole matrix element; such spin-orbit induced couplings are small enough to neglect under the conditions of interest here.

This allows us to make several approximations. Firstly, we can use non-relativistic potentials and transition dipole moments and in the calculation of the dynamic polarizability only consider singlet ${ }^{1} \Sigma^{+}$and ${ }^{1} \Pi$ potentials. Secondly, assuming a large detuning (such that $\left|E_{\gamma}-E_{v \tilde{J} M}-h \nu\right| \gg E_{v J M}$ for all states of interest) we can neglect the electric field and rotational dependence of the energy denominators in Eq. (4). Finally, we find that in the near infrared the contribution to the polarizability from intermediate states $\gamma$ in the ground $\mathrm{X}^{1} \Sigma^{+}$state is small. 
With this in mind the polarizability becomes

$$
\begin{aligned}
\alpha_{\sigma \sigma^{\prime}}^{v \tilde{J} M, \pm}(\nu) \cong \sum_{J, J^{\prime}} U_{\tilde{J}, J}^{v|M|} U_{\tilde{J}, J^{\prime}}^{v|M|} \sum_{e v_{e} \Lambda}\left\{\frac{1}{E_{e v_{e} \Lambda}-E_{v}+h \nu}+\frac{1}{E_{e v_{e} \Lambda}-E_{v}-h \nu}\right\} \\
\left.\times \sum_{J_{e} M_{e}} z v J M, \pm\left|d_{\sigma}\right| e v_{e} J_{e} M_{e} \Lambda\right\rangle_{z z}\left\langle e v_{e} J_{e} M_{e} \Lambda\left|d_{\sigma^{\prime}}\right| v J^{\prime} M, \pm\right\rangle_{z}
\end{aligned}
$$

where the energy $E_{v}$ is a typical vibrational energy in the ground state potential, and the rovibrational wavefunctions $\left|e v_{e} J_{e} M_{e} \Lambda\right\rangle$ of the electronically excited states with approximate energy $E_{e v_{e} \Lambda}$ are given by

$$
\left|e v_{e} J_{e} M_{e} \Lambda\right\rangle_{z} \equiv\left|e v_{e} \Lambda\right\rangle \times\left|J_{e} M_{e} \Lambda\right\rangle_{z} \equiv\left\{\frac{\phi_{v_{e}}(R)}{R}\left|e^{1} \Lambda\right\rangle\right\} \times\left\{\sqrt{\frac{2 J+1}{4 \pi}} D_{M_{e} \Lambda}^{J_{e}}(\hat{R})\right\}
$$

where the vibrational and electronic dependence has been isolated in the $\operatorname{ket}\left|e v_{e} \Lambda\right\rangle$ and the rotational dependence in $\left|J_{e} M_{e} \Lambda\right\rangle_{z}$, respectively. The wavefunction $\phi_{v_{e}}(R)$ is the radial rovibrational wavefunction, the ket $\left|e^{1} \Lambda\right\rangle$ is the electronic state with projection quantum number $\Lambda$ defined along the internuclear axis, and $D_{M M^{\prime}}^{J}(\hat{R})$ is a Wigner rotation matrix that describes a symmetric top rotational wavefunction. The sum over $\Lambda$ includes both positive and negative values, where $\Lambda=0$ corresponds to excited ${ }^{1} \Sigma^{+}$electronic states and $\Lambda= \pm 1$ to ${ }^{1} \Pi$ states.

As stated before the energy of the excited state and $\phi_{v_{e}}(R)$ depend on the electronic state $\left|e^{1} \Lambda\right\rangle$ and vibrational level $v_{e}$, but not $J_{e}$ and $M_{e}$. Consequently, the transition dipole moments separate into $z\left\langle v J M, \pm\left|d_{\sigma}\right| e v_{e} J_{e} M_{e} \Lambda\right\rangle_{z}=\left\langle v J|d(R)| e v_{e} \Lambda\right\rangle F_{J M \pm, \sigma}^{J_{e} M_{e} \Lambda}$, where $F_{J M \pm, \sigma}^{J_{e} M_{e} \Lambda}$ is an integral of the product of three Wigner rotation matrices $D_{M M^{\prime}}^{J}(\hat{R})$ over the orientation of the molecule $\hat{R}$ that can be evaluated using angular momentum algebra [20].

Moreover, we have verified that $\left\langle v J|d(R)| e v_{e} \Lambda\right\rangle$ is nearly independent of $J$. The sums over $J_{e}$ and $M_{e}$ in Eq. (5) can now be performed and we finally find

$$
\begin{aligned}
\left\langle v \tilde{J} M, \pm\left|\alpha_{\sigma \sigma^{\prime}}(\nu)\right| v \tilde{J} M, \pm\right\rangle & = \\
\sum_{\Lambda} \alpha^{\Lambda}(\nu) \sum_{J, J^{\prime}} U_{\tilde{J}, J}^{v|M|} U_{\tilde{J}, J^{\prime}}^{v|M|} & \times \sum_{J_{e} M_{e}} F_{J M \pm, \sigma}^{J_{e} M_{e} \Lambda} F_{J^{\prime} M \pm, \sigma^{\prime}}^{J_{e} M_{e} \Lambda}
\end{aligned}
$$

with the $\Lambda$-dependent

$$
\alpha^{\Lambda}(\nu)=\sum_{e v_{e}}\left\langle v J|d| e v_{e} \Lambda\right\rangle\left\langle e v_{e} \Lambda|d| v J\right\rangle\left\{\frac{1}{E_{e v_{e} \Lambda}-E_{v}+h \nu}+\frac{1}{E_{e v_{e} \Lambda}-E_{v}-h \nu}\right\} .
$$

The parallel $\alpha^{0}(\nu)$ and perpendicular $\alpha^{1}(\nu)$ contributions to the polarizability are due to transitions to the $\Sigma$ and $\Pi$ states, respectively.

\section{Magic DC Electric Field}

The polarizability depends on the stength of an external electric field through the use of the unitary matrices $U_{\tilde{J}, J}^{v M}$ of Eq. (6). Figure 2 shows the dynamic polarizability of the ground states of the KRb and RbCs molecules as a function of the external electric field strength. The left panels of these figures correspond to the parallel $(\vec{\epsilon}=\hat{z})$ and 

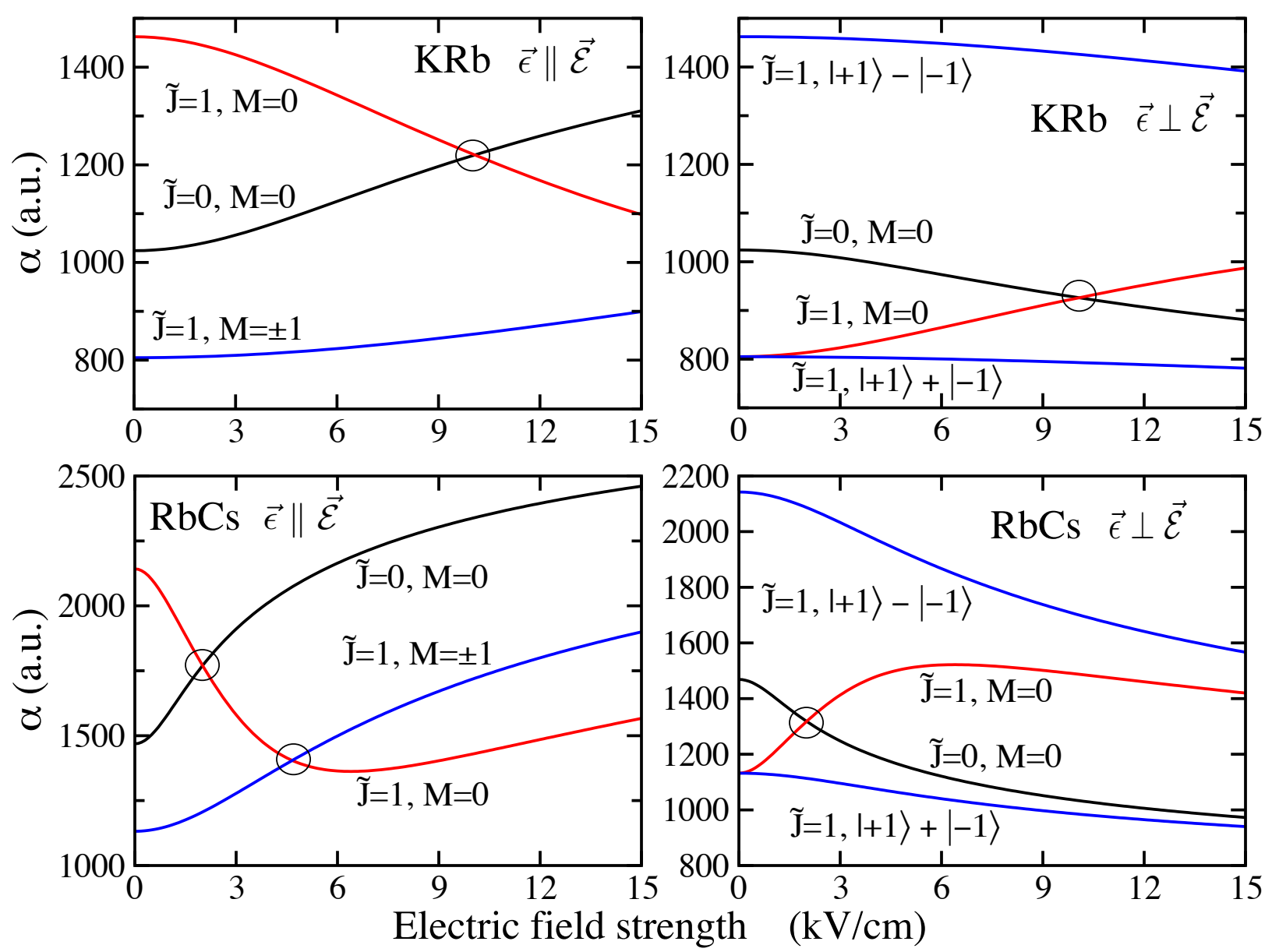

FIG. 2: Dynamic polarizability at a wavenumber of $9174 \mathrm{~cm}^{-1}$ of the $v=0, \tilde{J}=0$ and 1 levels of the $\mathrm{X}^{1} \Sigma^{+}$ground state of $\mathrm{KRb}$ (top row) and RbCs (bottom row) as a function of the field strength of an external electric field. The polarization of the trapping field is parallel (left panel) and perpendicular (right panel) to the direction of the electric field. The circles indicate a crossing point between $\tilde{J}=0$ and 1 polarizabilities. The degeneracy of the states $\tilde{J}=1$ and $M= \pm 1$ is lifted by perpendicularly polarized laser light. The new states are labeled $|+1\rangle \pm|-1\rangle$, corresponding to $|\tilde{J}=1, M \pm\rangle$ respectively. The $M= \pm 1$ states remain degenerate for parallel polarization.

the right panels to the perpendicular $(\vec{\epsilon}=\hat{x})$ polarization of the trapping light relative to the electic field direction $\overrightarrow{\mathcal{E}}$. In all cases the polarizability depends on both the state index $\tilde{J}$ and the angular momentum projection $M$. For KRb (Fig. 2, top row) a "magic" electric field strength exists at $\mathcal{E}=10 \mathrm{kV} / \mathrm{cm}$, where the polarizability of $\tilde{J}=0, M=0$ and $\tilde{J}=1, M=0$ states coincide. This is possible due to the polar character of the molecules. For RbCs (Fig. 2, bottom row) two "magic" electric field strengths exist. For the pair of states $\tilde{J}=0, M=0$ and $\tilde{J}=1, M=0$ a crossing occurs near $2 \mathrm{kV} / \mathrm{cm}$. Another crossing appears for the states $\tilde{J}=1, M=0$ and $\tilde{J}=1, M= \pm 1$ at $4.7 \mathrm{kV} / \mathrm{cm}$. They both are at a much smaller field strength than for KRb, since RbCs has a larger permanent dipole moment and smaller rotational splittings in the ground state. Note that the $M=0$ "magic" electric field occurs at the same field strength for both parallel and perpendicular polarization of the trapping light. In fact, they are the same for any polarization. 


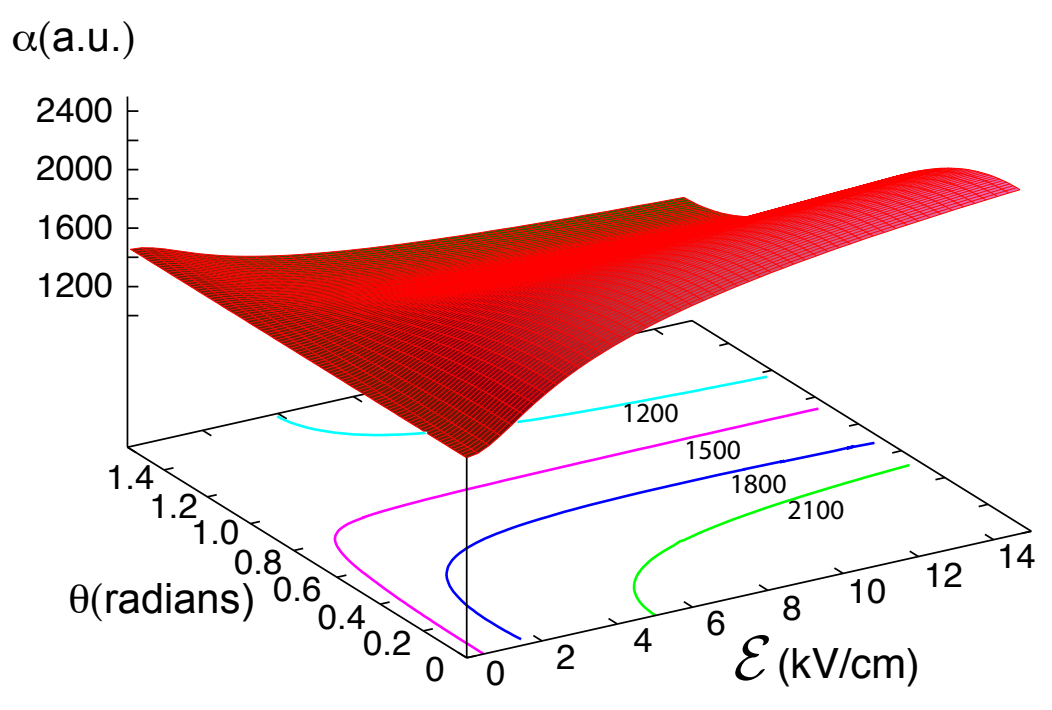

FIG. 3: Dynamic polarizability of the $v=0, \tilde{J}=0, M=0$ level of the $\mathrm{X}^{1} \Sigma^{+}$ground state of RbCs as a function of external electric field strength and the angle $\theta$ between the direction of the electric field $\overrightarrow{\mathcal{E}}$ and the polarization $\vec{\epsilon}$ of the trapping light at a wavenumber of $9174 \mathrm{~cm}^{-1}$. The contour lines are marked by the polarizability value in atomic units.

\section{Magic Angle}

In future experiments one would expect to be able to change the angle between the static and dynamic electric fields. Figure 3 shows a surface plot of the dynamic polarizability as a function of $\mathcal{E}$ and the angle $\theta$ between $\overrightarrow{\mathcal{E}}$ and polarization of the trapping light $\vec{\epsilon}$, for linearly polarized light. The polarizability depends smoothly on both $\theta$ and $\mathcal{E}$.

Figure 4 compares the dynamic polarizability of the states $|\tilde{J}=0\rangle$ and $|\tilde{J}=1, M=0, \pm 1\rangle$ as a function of the angle $\theta$ of the linear polarization of the optical field relative to the static field (such that $\hat{\epsilon}=\cos \theta \hat{z}+\sin \theta \hat{x}$ ), for several static electric field strengths within the range from 0 to $6 \mathrm{kV} / \mathrm{cm}$ for $\mathrm{KRb}$ and 0 to $3 \mathrm{kV} / \mathrm{cm}$ for RbCs. All curves with $M=0$ cross at the angle $\theta=\theta_{0}$ such that $\cos ^{2} \theta_{0}=1 / 3$, or $\theta_{0} \approx 54$ degrees. We refer to this as the "magic angle" since here the AC Stark shift is independent of the internal state of the molecule. This behavior occurs in many contexts and is a simple consequence of the rank- 2 tensor structure of the polarizability [24]. The angular dependence of the $\tilde{J}=0$ level is smaller than that of the $\tilde{J}=1$ levels as expected. It inherited the zero-electric field properties of the scalar $J=0$ state. As seen in Fig. 2, the RbCs polarizability of the $\tilde{J}=0, M=0$ and $\tilde{J}=1, M=0$ levels cross at a magic electric field strength of $2 \mathrm{kV} / \mathrm{cm}$. For Fig. 4 this implies that the curves start to "overlap" when $\mathcal{E}$ is equal or larger than this magic field value.

The polarizability operator $\alpha_{\sigma \sigma^{\prime}}(\nu)$, defined by Eq. (4), is a reducible rank-two tensor operator. Hence it can be expressed as a sum of irreducible tensor operators $\alpha^{(k)}(\nu)$ of rank $k=0,1,2$. In terms of these irreducible tensor operators, the AC Stark shift $\Delta E$ is 

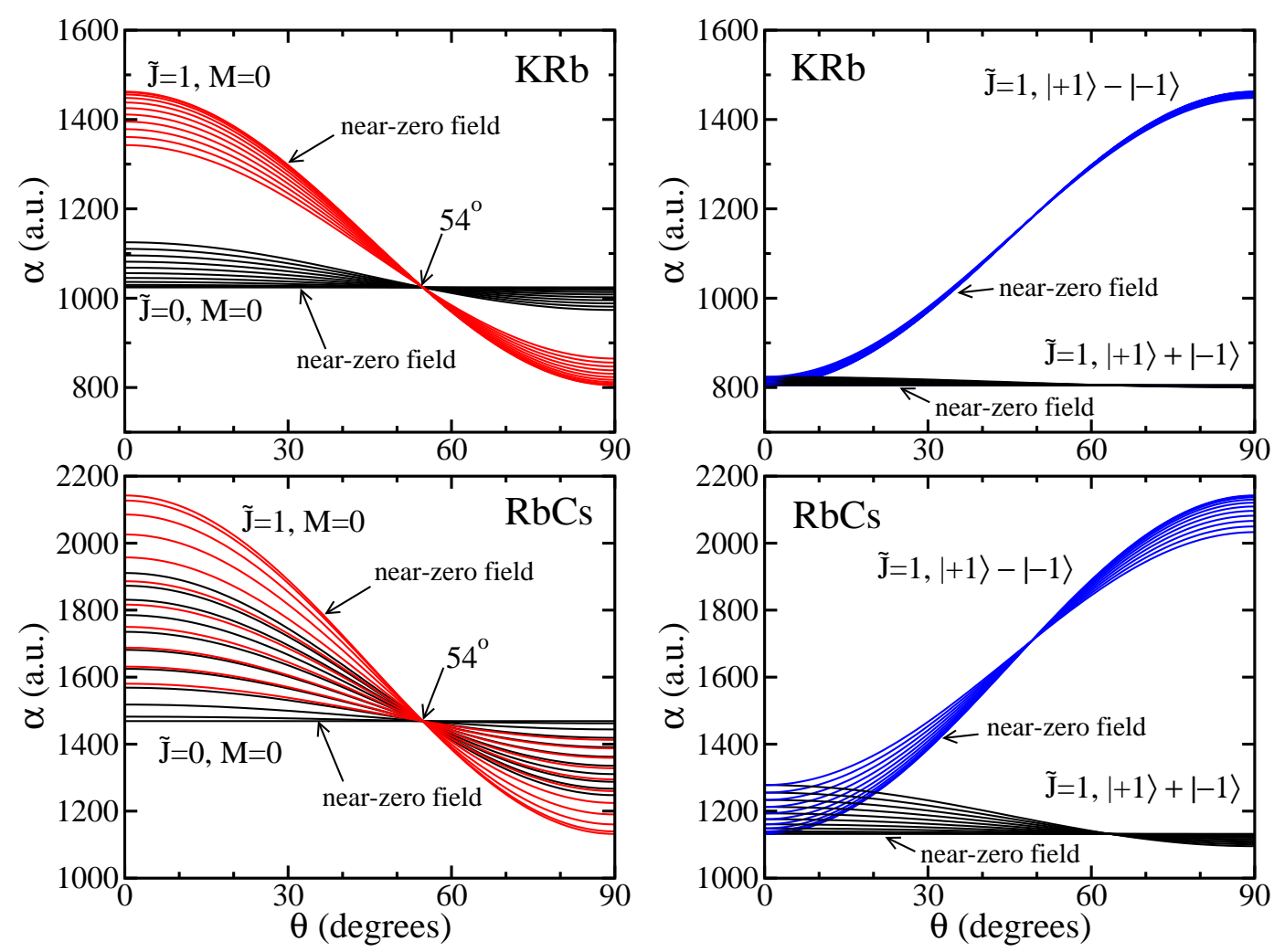

FIG. 4: Dependence of the dynamic polarizability on the angle between the polarization $\vec{\epsilon}$ of the trapping light at a wavenumber of $9174 \mathrm{~cm}^{-1}$ and the direction of the external electric field $\overrightarrow{\mathcal{E}}$, for different values of $\mathcal{E}$. The polarizability of states with projections $M=0$ and $M= \pm 1$ of the $\mathrm{X}^{1} \Sigma^{+}$ ground state of $\mathrm{KRb}$ and $\mathrm{RbCs}$ are shown in the left and right panels, respectively. For clarity $\mathcal{E}$ ranges from $0.6 \mathrm{kV} / \mathrm{cm}$ to $6 \mathrm{kV} / \mathrm{cm}$ in steps of $0.6 \mathrm{kV} / \mathrm{cm}$ for $\mathrm{KRb}$ and from $0.3 \mathrm{kV} / \mathrm{cm}$ to 3 $\mathrm{kV} / \mathrm{cm}$ in steps of $0.3 \mathrm{kV} / \mathrm{cm}$ for RbCs. At the "magic angle" $\theta=\theta_{0}=54^{\circ}$, the polarizability of the states $\tilde{J}=0, M=0$ and $\tilde{J}=1, M=0$ are identical and independent of the strength of the static electric field. For the $\tilde{J}=1, M= \pm 1$ states no magic angle exists.

proportional to the diagonal matrix element of the operator $\sum_{\sigma \sigma^{\prime}} \alpha_{\sigma \sigma^{\prime}}(\nu) \epsilon_{\sigma} \epsilon_{\sigma^{\prime}}^{*}$, which can be written in the general form

$$
\sum_{k=0}^{2} \sum_{q=-k}^{k}(-1)^{q} \alpha_{q}^{k}(\nu) \epsilon \epsilon_{-q}^{k},
$$

where $q$ is a spherical tensor projection index and the explicit forms of the irreducible spherical tensors $\alpha_{q}^{k}(\nu)$ and $\epsilon \epsilon_{q}^{k}$ are given in Refs [21, 22].

In order to derive the "magic angle" condition for $M=0$ states, we consider the effect of each term in the expansion (8). The term with $k=0$ corresponds to the scalar polarizability; the operator $\alpha_{0}^{0}(\nu)=\sum_{\sigma \sigma^{\prime}} \alpha_{\sigma \sigma^{\prime}}(\nu) \delta_{\sigma \sigma^{\prime}}$ has, under our approximations, diagonal matrix elements that are independent of $J$ ( or $\tilde{J}$ ) and $M$ for all states of interest, and similarly the quantity $\epsilon \epsilon_{0}^{0} \propto \vec{\epsilon} \cdot \vec{\epsilon}^{*}=1$ is independent of the polarization of the optical field. The term with $k=1$, corresponding to the vector polarizability, in general is significant. However, for the special case of linearly polarized light where $\vec{\epsilon}$ is real, the quantities $\epsilon \epsilon_{q}^{1}=\left(\vec{\epsilon} \times \vec{\epsilon}^{*}\right)_{q}$ vanish and hence the effect of the vector polarizability is zero. For the tensor polarizability 
terms (with $k=2$ ), from the Wigner-Eckhart theorem only the operator component with $q=0$ gives rise to a non-zero diagonal matrix element for $M=0$ states. Hence the contribution of this term is proportional to $\epsilon \epsilon_{0}^{2}$. Without loss of generality we can define the linear polarization as $\vec{\epsilon}=\epsilon_{x} \hat{x}+\epsilon_{z} \hat{z}=\cos \theta \hat{z}+\sin \theta \hat{x}$. In this case $\epsilon \epsilon_{0}^{2} \propto \epsilon_{z} \epsilon_{z}^{*}-\vec{\epsilon} \cdot \vec{\epsilon}^{*} / 3=\cos ^{2} \theta-1 / 3$. Hence the contribution to $\Delta E$ due to the tensor polarizability also vanishes for all states with $M=0$, when the optical field is linearly polarized at the "magic angle" $\theta=\theta_{0}$. Under this condition the only contribution to the dynamic polarizability is from the $k=0$ scalar term, which is the same for all states of interest.

The property of the "magic" electric field discussed in Fig. 2 can also be understood in terms of the tensor structure of the polarizability. At certain values of the applied DC electric field $\mathcal{E}$, the rank-2 components of the polarizability, $\alpha_{0}^{2}$, of the $\tilde{J}=0, M=0$ and $\tilde{J}=1, M=0$ levels becomes the same. When this condition is met, the AC Stark shift becomes independent of the direction of the trapping light's linear polarization.

Here we finish with an example of a specific implementation of a "magic angle" 3-D lattice. Let the lattice be formed by three orthogonal retroreflected laser beams $a, b$, and $c$, with initial propagation directions $\hat{k}$ given by $\hat{k}_{a}=\hat{y}, \hat{k}_{b}=(\hat{x}+\hat{z}) / \sqrt{2}$, and $\hat{k}_{c}=(\hat{x}-\hat{z}) / \sqrt{2}$. These three beams each have a different frequency $\nu$, such that $\nu_{a}=\nu_{b}-\delta_{b}=\nu_{c}-\delta_{c}$; here the offset frequencies $\delta_{b, c}$ must satisfy $\nu_{a, b, c} \gg \delta_{b, c} \gg f_{m o t}$, where $f_{m o t}$ is the motional frequency of the molecules in the optical trapping potential. The use of different frequencies (which can be generated from a single laser by using e.g. acousto-optic modulators) in this manner eliminates the effect of interference terms between the different laser beams: such terms average to zero rapidly over the time of motion of the atom, and hence can be neglected. The resulting average trap potential is then simply the sum of the potentials due to each individual laser beam. Finally, the polarizations of the three beams can be chosen

as $\hat{\epsilon}_{a}=\sqrt{2 / 3} \hat{x}+\sqrt{1 / 3} \hat{z} ; \hat{\epsilon}_{b}=\sqrt{2 / 3} \hat{k}_{c}+\sqrt{1 / 3} \hat{y} ;$ and $\hat{\epsilon}_{c}=\sqrt{2 / 3} \hat{k}_{b}+\sqrt{1 / 3} \hat{y}$. In each case, $|\hat{\epsilon} \cdot \hat{z}|=\cos \theta_{0}$.

[1] J. Ye, H. J. Kimble, and H. Katori, Science 320, 1734 (2008).

[2] H. Katori, M. Takamoto, V. G. Pal'chikov, V. D. Ovsiannikov, Phys. Rev. Lett. 91, 173005 (2003).

[3] V. V. Flambaum, V. A. Dzuba, and A. Derevianko, Phys. Rev. Lett. 101, 220801 (2008).

[4] K. Beloy, A. Derevianko, V. A. Dzuba, and V. V. Flambaum, Phys. Rev. Lett. 102, 120801 (2009).

[5] N. Lundblad, M. Schlosser, and J. V. Porto, Phys. Rev. A 81, 031611(R) (2010).

[6] A. Derevianko, Phys. Rev. A 81, 051606(R) (2010).

[7] J. Doyle, B. Friedrich, R. V. Krems, and F. Masnow-Seeuws, Special issue on ulatracold polar molecules, Eur. Phys. J. D 31, 149 (2004).

[8] L.D. Carr, D. DeMille, R.V. Krems, Jun Ye, New J. Phys. 11, 055049 (2009).

[9] D. DeMille, Phys. Rev. Lett. 88, 067901 (2002).

[10] R. Zhao, el al. Nat. Phys. 5, 100 (2009).

[11] T. Zelevinsky, S. Kotochigova, and Jun Ye, Phys. Rev. Lett. 100, 043201 (2008).

[12] S. Kotochigova, T. Zelevinsky, and Jun Ye, Phys. Rev. A 79, 012504 (2009). 
[13] S. Ospelkaus, K.-K. Ni, M. H. G. de Miranda, B. Neyenhuis, D. Wang, S. Kotochigova, P. S. Julienne, D. S. Jin, and J. Ye, Faraday Discuss. 142, 351 (2009).

[14] A. Micheli, G.K. Brennen, and P. Zoller, Nature Phys. 2, 341 (2006).

[15] H. P. Büchler, E. Demler, M. Lukin, A. Micheli, N. Prokof'ev, G. Pupillo, and P. Zoller, Phys. Rev. Lett. 98, 060404 (2007).

[16] H. P. Büchler, A. Micheli, and P. Zoller. Nature Phys. 3, 726 (2007).

[17] S. Kotochigova and E. Tiesinga, Phys. Rev. A 73, 041405(R) (2006).

[18] S. Kotochigova, E. Tiesinga, P. S. Julienne, New J. Phys. 11, 055043 (2009).

[19] S. Kotochigova, New J. Phys. 12, 073041 (2010).

[20] D. M. Brink and G. R. Satchler, Angular momentum, (Clarendon Press, London, 1993).

[21] K.D. Bonin and V.V. Kresin, Electric-dipole polarizabilities of atoms, molecules, and clusters, (World Scientific, Singapore, 1997).

[22] D.A. Varsholovich, A.N. Moskalev, and V.K. Khersonskii, Quantum theory of angular momentum: irreducible tensors, spherical harmonics, vectors coupling coefficients, 3nj symbols, (World Scientific, Singapore, 1988).

[23] A. J. Stone, The theory of intermolecular forces, (Clarendon Press, London, 1996).

[24] D. Budker, D.F. Kimball, and D.P. DeMille, Atomic Physics: an exploration through problems and solutions, $2^{\text {nd }} e d .$, (Oxford Univ. Press, Oxford, 2008).

\section{ACKNOWLEDGMENTS}

This work is supported by a MURI grant of the Air Force Office of Scientific Research; by NSF; and (for DD) by DOE. SK acknowledges helpful discussions with J. Ye, D. Jin, and B. Neyenhuis. 